Documents pour l'histoire des techniques

Nouvelle série

$17 \mid 1^{\text {er }}$ semestre 2009

L'invention technique et les figures de l'inventeur (XVIII $-X^{\mathrm{e}}$ siècles)

\title{
L'évolution des techniques photographiques de prise de vue (1839-1920). Mise en évidence d'un système sociotechnique
}

The evolution of photographic (1839-1920). The show case of a socio-technical system

\section{Marie-Sophie Corcy}

\section{OpenEdition}

Journals

Édition électronique

URL : http://journals.openedition.org/dht/494

DOI : $10.4000 /$ dht.494

ISSN : 1775-4194

Éditeur :

Centre d'histoire des techniques et de l'environnement du Cnam (CDHTE-Cnam), Société des élèves du CDHTE-Cnam

\section{Édition imprimée}

Date de publication : 31 mars 2009

Pagination : 57-68

ISBN : 978-2-95-30779-3-3

ISSN : 0417-8726

\section{Référence électronique}

Marie-Sophie Corcy, «L'évolution des techniques photographiques de prise de vue (1839-1920). Mise en évidence d'un système sociotechnique », Documents pour l'histoire des techniques [En ligne], $17 \mid 7^{\text {er }}$ semestre 2009, mis en ligne le 27 septembre 2010, consulté le 07 septembre 2020. URL : http:// journals.openedition.org/dht/494; DOI : https://doi.org/10.4000/dht.494 


\title{
L'évolution des techniques photographiques de prise de vue (1839-1920) \\ Mise en évidence d'un système sociotechnique
}

\author{
Marie-Sophie Corcy \\ Musée des arts et métiers-Cnam - CDHTE-Cnam
}

\begin{abstract}
Résumé
L'instrument de la photographie, chambre ou appareil, se compose de différents éléments (châssis, obturateur, objectif), dont la caractéristique, outre celle de remplir chacun une fonction définie, réside dans leur aspect combinatoire. L'identification et l'analyse de corpus de données inédites (brevets, marques, ouvrages, articles communications), sur la période 1839-1920, nous ont permis de mettre en évidence la dynamique de l'évolution de ce "micro-système technique ", les phases et les caractéristiques de l'innovation, mais aussi les interactions entre ce système et d'autres connexes (optique, mécanique, chimie) ou périphériques (applications scientifiques ou industrielles).

Nous avons identifié les acteurs de l'innovation qui ont participé au processus d'industrialisation de la photographie, et les vecteurs de transmission des savoirs, soit le passage de l'invention à l'innovation, derrière lesquels apparaissent les stratégies de valorisation de l'invention. En effet, le choix de publier un ouvrage, de déposer brevet ou marque, ou de communiquer dans une société savante, révèle, chez les constructeurs, des manœuvres différentes du point de vue de la protection de l'invention mais aussi de sa diffusion.
\end{abstract}

Résumés et mots clés en anglais sont regroupés en fin de volume, accompagnés des mots clés français

L appareil photographique est un instrument répondant à une fonctionnalité précise. Composé de différents éléments (le châssis, la chambre noire ou corps de l'appareil, l'objectif, l'obturateur), il permet, par leur association rationnelle, de réaliser une image dite photographique. Ces éléments mettent en œuvre le principe physique optique (la convergence des rayons lumineux au fonds de la camera obscura) et le principe chimique (l'impression de l'image formée par les rayons lumineux et projetée sur une surface sensibilisée), nécessaires à la réalisation de cet acte technique. On peut d'ores et déjà constater que le processus photographique n'est pas une technique autonome, en ce sens qu'il regroupe et met en jeu des connaissances, savoir-faire et techniques affluentes (telle la chimie, l'optique ou la mécanique) empruntant à diverses sphères scientifiques et industrielles.

Cette combinaison technique correspond à la mise en œuvre d'un ensemble cohérent de structures compatibles les unes avec les autres, et peut s'apparenter en cela à l'idée de «système technique ». L'ob- jet de nos recherches était d'en faire la démonstration et de déterminer quelle était la dynamique de l'évolution de ce "micro-système technique " '. La période envisagée dans cette étude, de 1839 à 1920, se justifie par le choix de la date de la divulgation du procédé daguerréotype, et la fin de la Première Guerre mondiale, qui marque la mise en place presque définitive des techniques photographiques argentiques sur le plan de l'instantanéité et des applications.

Nous identifierons enfin les acteurs de l'innovation, qui ont participé au processus d'industrialisation de la photographie, et les vecteurs de transmission des savoirs, derrière lesquels apparaissent les stratégies de valorisation de l'invention.

1 Marie-Sophie Corcy, "Techniques photographiques de prise de vue, formes et applications scientifiques et industrielles de la photographie noir et blanc en France (18391920) 11, doctorat de l'université de Paris IV-Sorbonne, sous la direction de François Caron, 7 volumes, 1997, 1914 p. 


\section{L'évolution des techniques photographiques}

\section{Définition d'un corpus de sources : entre droits exclusifs et pensée collective}

Pour tenter de répondre à notre questionnement, nous avons identifié et exploité les brevets et les marques déposés en France concernant la photographie. Les brevets ont été répertoriés et quantifiés depuis 1839 dans la classe 12 « Instruments de précision », la classe 14 "Produits chimiques », et la classe 17 « Beauxarts " puis " Arts industriels " ${ }^{2}$. Ces dépôts, au nombre de 4228 (777 brevets sont déposés de 1839 à 1879 , 3451 de 1880 à 1920) permettent d'appréhender une tendance en termes d'émergence et de développement et en cela de mesurer l'innovation.

Les marques ont été répertoriées et quantifiées depuis 1858, dans la classe 43 «Instruments de musique et de précision », la classe 57 «Photographie et lithographie », et la classe 59 «Produits chimiques ». En attestant de la commercialisation, ces dépôts, au nombre de 3777 (58 marques ont été déposées de 1858 à 1879 et 3719 de 1880 à 1920), confirment l'innovation et mettent en évidence la phase d'industrialisation de la photographie.

Nous avons confronté ces données avec l'étude des articles et communication du journal La Lumière puis du Bulletin de la Société française de photographie, reflets de l'activité de la Société héliographique, puis de la Société française de photographie. Ces organes ont opéré une sélection parmi les inventions nouvelles et assuré la promotion d'innovations. Ils reflètent la vision des inventeurs et praticiens.

La Société héliographique fut la première société de photographie. Elle a été fondée en 1851 par des personnalités artistiques et scientifiques, telles qu'Eugène Delacroix, Benjamin Delessert, Victor Regnault, Edmond Becquerel, Hippolyte Bayard, Jean-Baptiste Louis Gros, Olympe Aguado, Edouard Baldus, Jules Champfleury, Charles Chevalier, Eugène Durieux, Gustave Le Gray, Henri Le Secq, Abel Niepce de Saint-Victor, ou Francis Wey. Les statuts, rédigés le 4 janvier 1851 par les membres du comité d'initiative, publiés le 9 février 1851 dans La Lumière, révèlent que « les séances de la Société [n'étaient] autre chose en principe, qu'une réunion agréable où la causerie [permettait] à chacun de choisir ses interlocuteurs, et où les sociétaires [étaient] des invités ${ }^{3}$. Mais l'impact de la revue fut considérable dans la diffusion de la connaissance. Francis Wey proposait en 1851 dans son éditorial un « exposé sommaire du but et des divers éléments du journal ${ }^{4}{ }^{4}$, fort révélateur et qui

2 L'entrée « photographie « apparaît dans la classification de 1863. 3 Article 13, La Lumière, 1851, p. 1, Marseille, rééd. Jeanne Laffitte, 1996, 2 vol.

4 Francis Wey, "Editorial », La Lumière, 1851, p. 17, Marseille, rééd. Jeanne Laffitte, 1996, 2 vol. permet déjà d'appréhender et de mesurer son impact sur l'enchaînement des progrès photographiques :

« Principalement consacré aux intérêts de l'héliographie, dont il a pour mission d'accélérer les progrès, le journal La Lumière rendra compte des inventions relatives au daguerréotype ou à la photographie ainsi que des diverses méthodes employées soit en France, soit en Angleterre, en Allemagne, ou en Amérique où les procédés sont fort avancés et où l'on entretiendra d'actives correspondances $॥$.

Il ajoutait :

"Les photographes les plus experts nous préparent des notes, au moyen desquelles on pourra suivre, sans maître et pas à pas, les manipulations nécessaires à la reproduction de la nature, au moyen de clichés de verre ou de papier $"$.

Antoine François Jean Claudet allait plus loin dans cette analyse ${ }^{5}$. Le journal était, selon lui, destiné « à exercer une influence considérable sur les travaux de la photographie ॥. II était destiné à cadrer les recherches, à les officialiser, et à les diffuser, enfin « [à détruire] l'empirisme et [à repousser] l'ignorance, qui est toujours accompagnée de charlatanisme ». La Lumière avait un autre but, « permettre à chacun de donner ses idées, de réclamer quand il s'agit de priorité dans un procédé ou dans une invention, d'offrir de nouvelles théories, et de discuter celles qui ont été antérieurement reçues ". Notons que cette dernière idée, source potentielle de polémiques, ne fut pas retenue par le Bulletin de la Société française de photographie. En 1854, dix-sept des membres de la Société héliographique, dont ses fondateurs, la quittaient et fondaient la Société française de photographie. Victor Regnault en fut le premier président. Cette société, qui réunissait en conséquence les mêmes personnalités que la Société héliographique, s'inscrivait dans la continuité de cette dernière. La Société héliographique, puis la Société française de photographie, réunissait ainsi les amateurs de photographie. Elles accueillaient et encourageaient les recherches, favorisant une émulation favorable à l'avancement des procédés et techniques. La Société française de photographie publia, en 1855, un bulletin, rédigé sous la direction de ses membres, conformément à l'article 37 de ses statuts:

5 Antoine François Jean Claudet, "Correspondance ", La Lumière, 1851, p. 18, Marseille, rééd. Jeanne Laffitte, 1996, 2 vol. 


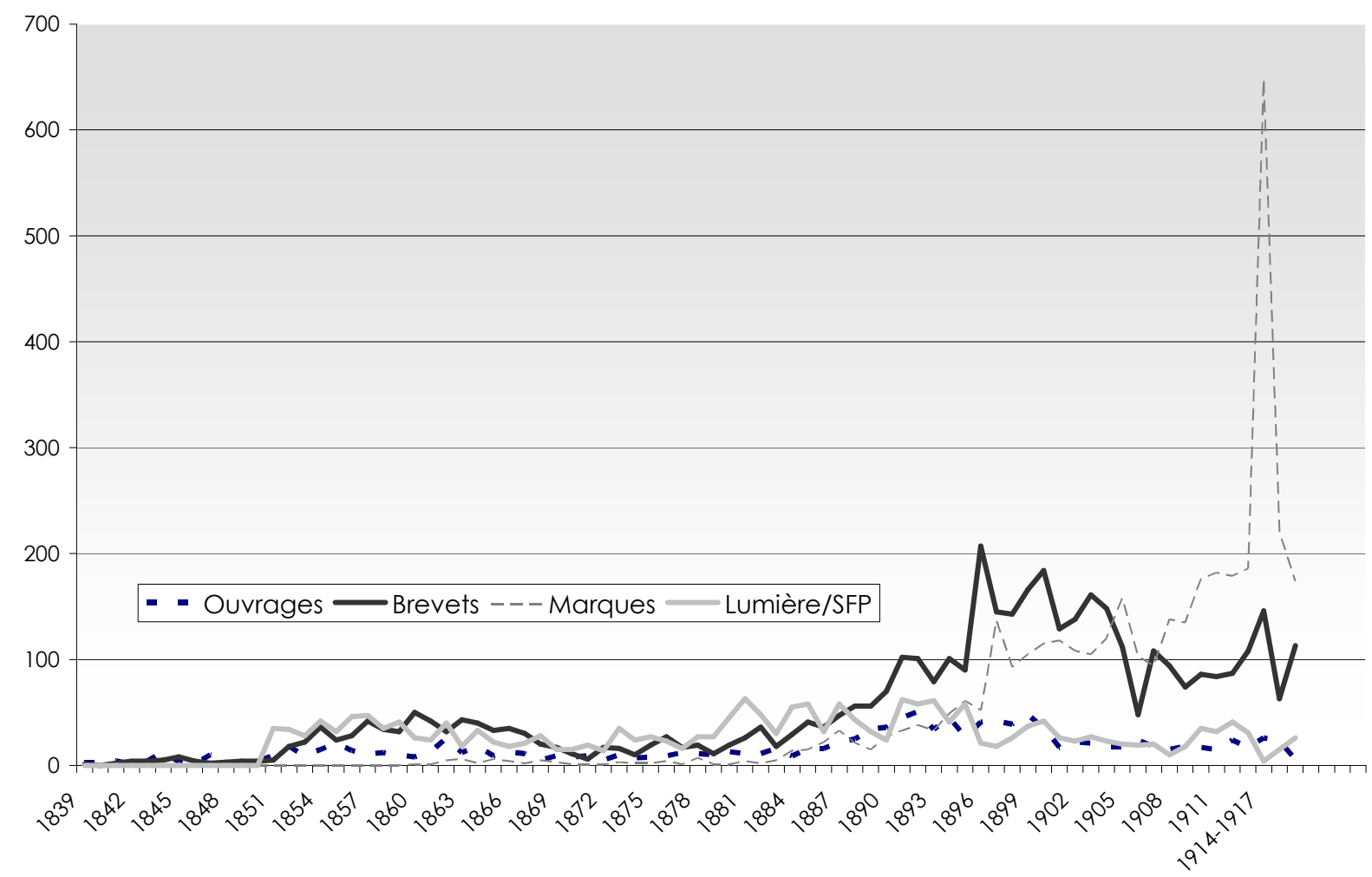

Fig. 1 - Étude statistique des publications

" La Société assurera, soit en publiant elle-même un recueil, soit en traitant à cet effet, avec un journal déjà existant, la publicité de ses travaux. Cette publicité comprendra, indépendamment des bulletins officiels des travaux de la Société, l'annonce et l'appréciation des nouvelles méthodes, des améliorations obtenues, des découvertes qui seraient faites, des manipulations qui faciliteraient les expériences, de tout ce qui peut, en un mot, servir au perfectionnement du dessin par la lumière. La publication sera, autant que possible, illustrée par des épreuves photographiques, choisies parmi celles qui seraient jugées intéressantes sous le rapport du résultat artistique ou curieuses sous le rapport de l'opération photographique $\|{ }^{6}$.

Ce bulletin fut en quelque sorte l'organe d'une pensée collective. II reproduisait les procès-verbaux et comptes rendus des séances, assemblées générales ou extraordinaires de la société. Dans une seconde partie, il rapportait au travers de notices scientifiques la description des procédés et appareils. Ainsi le choix de ces deux organes, La Lumière (appréhendé de 1851 à 1854) et du Bulletin de la Société française de photographie (de 1855 à 1920) se justifie par le rôle

6 Article 37, Bulletin de la Société française de photographie, Paris, Société française de photographie, 1855, p. 1. majeur de ces sources. Ces deux revues, porte-parole des scientifiques, des constructeurs et des praticiens, témoignent de l'incroyable activité inventive qui anima cette période. Ces sociétés savantes ont soutenu et filtré l'innovation.

Les communications pouvaient cependant se révéler surprenantes, comme l'atteste cette citation de l'opticien Charles Chevalier', issue d'un traité qu'il publia en 1854 :

" Lorsqu'on étudie l'histoire de la Photographie et que l'on cherche à se rendre compte des progrès que cet art fait chaque jour, on est surpris de la bizarrerie des combinaisons imaginées par les chercheurs [...]. ॥

Notons que, dans la majorité des cas et dans la tradition de la Société d'encouragement pour l'industrie nationale ${ }^{8}$, les communications étaient assor-

7 Charles Chevalier, Guide du photographe. Description et emploi raisonné des instruments d'optique appliqués à la photographie, Nouveaux mémoires et renseignements sur les moyens d'obtenir de belles épreuves sur papier, collodion, albumine et plaques métalliques par messieurs G. Roman, Cuvelier, Dufaur, Laborde, Arthur Chevalier, etc., Eloge de Daguerre, documents historiques, lettres inédites de N. Niepce, etc., Paris, Charles Chevalier, Paris, Charles Chevalier, 1854, p. 1. 8 Rappelons que le siège de la Société d'encouragement 


\section{L'évolution des techniques photographiques}

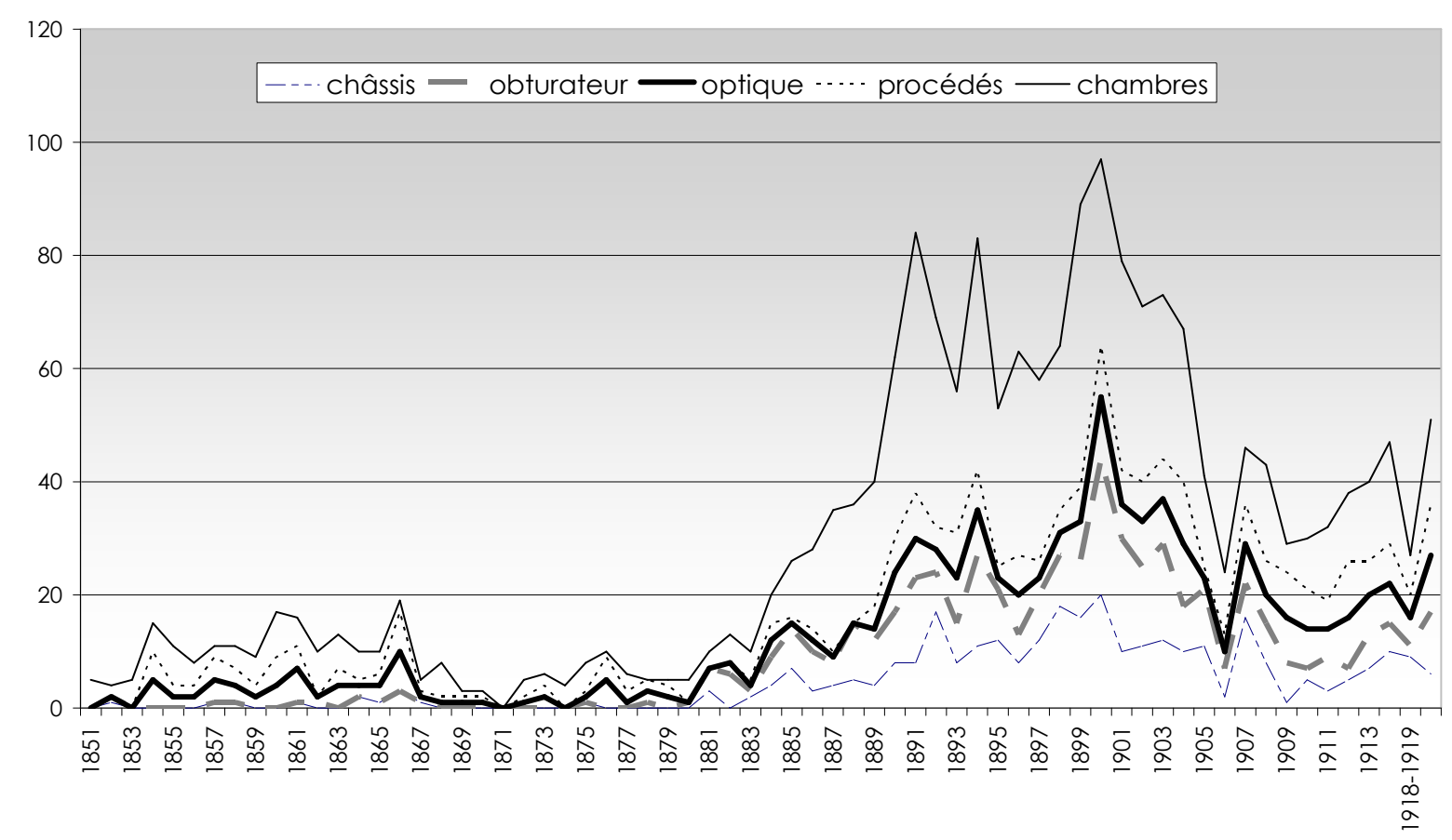

Fig. 2 - Brevets dans la photographie

ties de la présentation d'épreuves réalisées avec un procédé ou de l'appareil concerné, conformément à l'article 33 des statuts de la société'. Une partie de ces collections sera présentée en 1927 dans les nouvelles salles consacrées à la photographie et au cinéma au Conservatoire des arts et métiers.

Nous avons répertorié les traités de photographie publiés dès 1839. Près de trois mille ouvrages ont été localisés dans des bibliothèques parisiennes ${ }^{10}$, choisies pour la nature et l'importance de leur fonds photographique, et bien sûr à la Société française de photographie. Ces publications permettent d'appréhender les modes de développement des techniques dans un contexte général. Elles constituent un indicateur de la pénétration de la technique dans la société, et en cela informent sur la pratique du médium ou sur la mise en pratique d'une invention. Nous verrons

pour l'industrie nationale et celvi de la Société héliographique étaient tous deux abrités par l'Hôtel de l'industrie, place Saint-Germain-des-Prés à Paris et que ces deux sociétés savantes comportaient des membres communs.

9 " Toute communication d'un procédé nouveau devra, autant que possible, être accompagnée de quelques preuves ou de résultats matériels propres à confirmer par l'expérience l'exactitude de la théorie et la réalité du perfectionnement ", article 33, Bulletin de la Société française de photographie, Paris, Société française de photographie, 1855, p. 11. 10 Bibliothèque nationale de France, Bibliothèque historique de la Ville de Paris, Bibliothèque centrale du Conservatoire des arts et métiers, Société française de photographie. en effet (cf. fig. 1) que l'étude statistique portant sur les publications donne des résultats conformes à ceux révélés par les brevets et les marques, du point de vue de l'évolution des techniques photographiques. Le rythme des publications suit celui des dépôts de brevets et des dépôts de marques.

On doit cependant distinguer dans cette analyse les traités généraux de photographie des traités spécialisés dans l'une ou l'autre branche de la photographie, les traités à destination des praticiens avertis de ceux conçus pour les amateurs, les traités écrits par des anonymes de ceux publiés par des vulgarisateurs scientifiques reconnus.

\section{La dynamique de l'innovation}

Si l'on se situe du point de vue d'une approche globale, l'analyse statistique des dépôts de brevets et de marques, des publications, et des communications issues de La Lumière et du Bulletin de la Société française de photographie nous révèle plusieurs phénomènes :

- les interactions entre le système technique photographique et des systèmes connexes (optique, mécanique, chimie...) ;

- la mise en évidence d'innovations au départ des cycles de croissance (assimilés à des périodes de progrès technique) ; 


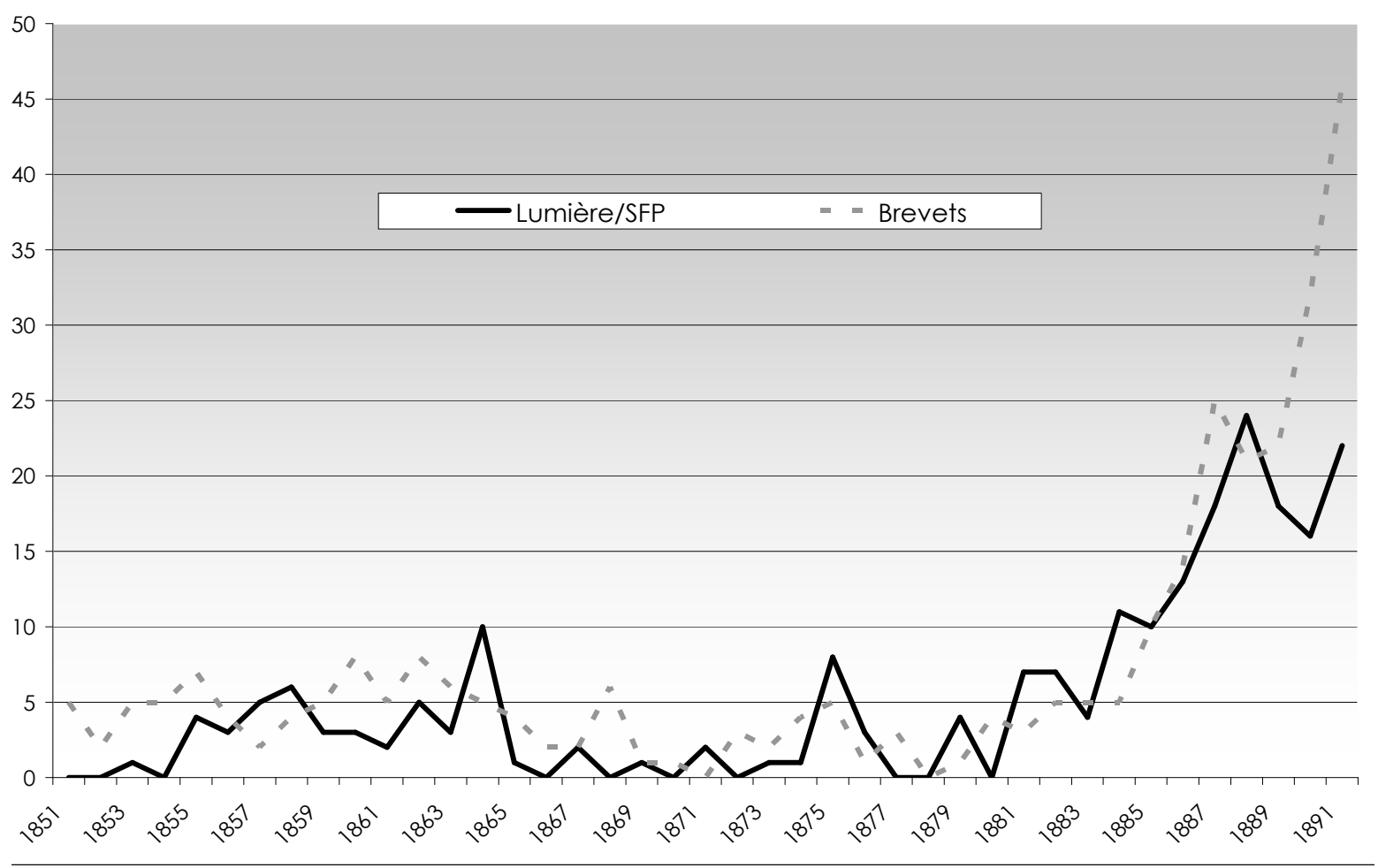

Fig. 3 - Chambres et appareils photographiques

- la différenciation entre des innovations dominantes de niveau $1^{11}$, insufflant une dynamique à l'ensemble du système technique et des innovations de niveau $2^{12}$, inhérentes à une technologie particulière, dont les conséquences ont été moindres sur le système technique mais dont l'apparition résulte d'innovations dominantes de niveau 1. Toutefois ces innovations de niveau 2 s'inscrivent dans une continuité technologique.

Nous avons également constaté la présence de «systèmes périphériques » (représentés par les formes, les applications scientifiques et industrielles de la photographie) au système technique générique, et leur constante interaction en terme de transfert technologique.

Nous avons croisé les données en fonction des typologies repérées (supports et procédés sensibles, éléments constitutifs de la chambre ou appareils photographiques). Ce recensement thématique a permis de mettre en évidence la logique de l'évolution de chacun de ces composants. Cette vision éclatée de

11 L'émulsion au gélatino-bromure d'argent.

12 Talbotype et calotype, procédé à l'albumine, collodion humide, collodion sec, émulsions orthochromatique et panchromatique au gélatino-bromure d'argent, verre, support celluloïd, objectif achromatique, objectif orthoscopique, objectif aplanétique, objectif rectilinéaire, objectif anastigmat, obturateur à guillotine, obturateur à volet, obturateur de plaque, obturateur central, etc. l'évolution technique de la chambre photographique permet de mettre en évidence la notion de « microsystème technique ॥, appliquée à la filière technique de la photographie.

On remarquera, l'aspect strictement combinatoire des éléments composant cet « ensemble de techniques $»$, et leur interdépendance, assurant la cohérence globale du système (en considérant que les chambres photographiques, les procédés sensibles, l'optique, les systèmes d'obturation et les châssis constituent respectivement une technologie et leur association le « système technique photographique générique ») (cf. fig. 2). On peut ainsi établir cette métaphore : la chambre photographique, rassemblement de ces éléments constitutifs, apparaît comme le microcosme du système technique photographique.

On pourra constater la cohérence entre les sources exploitées, qui montrent un mouvement d'évolution similaire, mais présentent quelquefois un décalage entre les phases de croissance et de décroissance. Ainsi, les dépôts de brevets et les communications dans les sociétés savantes concernant des chambres et appareils photographiques sont cohérents en termes de périodisation (cf. fig. 3).

Mais si l'on s'attache au développement des systèmes d'obturation, on constate que les recherches, souvent empiriques, sont présentées à la Société française de photographie avant de donner lieu à 


\section{L'évolution des techniques photographiques}

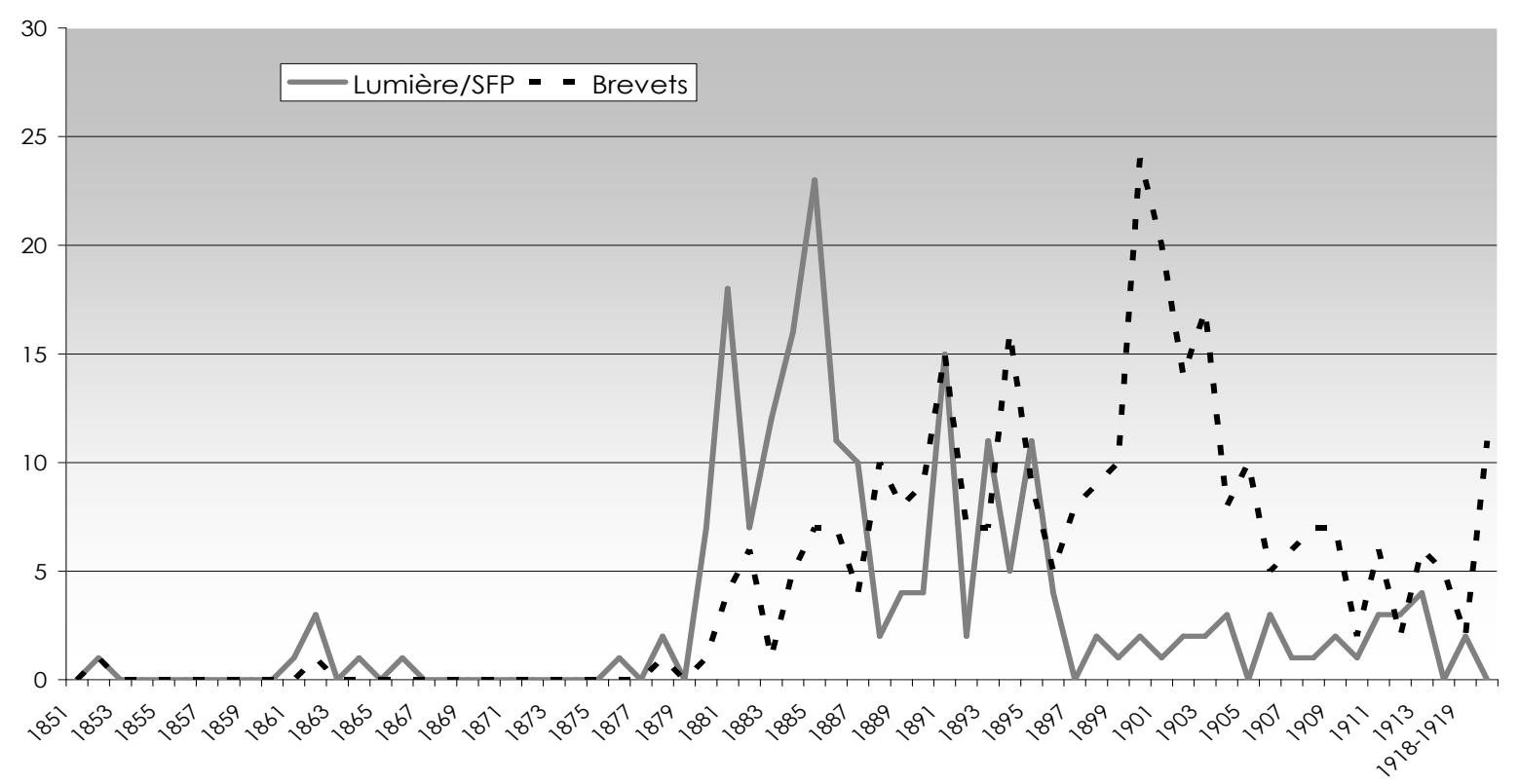

Fig. 4 - Systèmes d'obturation

un dépôt de brevet. L'analyse des données révèle le cheminement de l'invention et les stratégies des inventeurs (cf. fig. 4).

La différence quantitative du nombre des inventions en fonction de la thématique adoptée ${ }^{13}$ marque une hiérarchie entre ces techniques et fait entrevoir le moteur de l'évolution du système, à savoir les recherches en matière de sensibilité des procédés chimiques - recherches motivées par un besoin, celui de la diversification des usages potentiels ou latents de la photographie. La chimie est, depuis l'origine de l'invention, au cœur du système et elle en détermine l'évolution.

La chimie créant la dynamique du système, les recherches en la matière se révélèrent prédominantes. Cependant, elles n'apparaissent pas ou très peu à travers les dépôts de brevets (239 brevets de 1851 à 1920, soit 12\% de l'ensemble des dépôts) mais au travers des communications de la Société héliographique puis de la Société française de photographie (1079 communications identifiées de 1851 à 1920, soit près de $52 \%$ de l'ensemble des communications) et des dépôts de marques (908 dépôts de 1859 à 1920, soit près de $25 \%$ de l'ensemble des dépôts). Les recherches en matière de procédés sensibles, de châssis, de systèmes d'obturation et d'optique sont, d'un point de vue quantitatif, d'un volume équivalent concernant les dépôts de brevets (entre 12 et $16 \%$ de

13 Procédés sensibles, systèmes optiques, systèmes d'obturation, châssis. l'ensemble des dépôts). Les chambres ou appareils représentent $44 \%$ des brevets.

Le degré d'impressionnabilité des surfaces sensibles et par voie de conséquence la diminution du temps d'exposition nécessaire à la capture de l'image reçue à l'intérieur de la chambre noire, sont à l'origine du développement de la filière photographie ; ce qui provoqua, à mesure des progrès réalisés, une restructuration du système, caractérisée par l'adaptation successive des différents éléments constitutifs de la chambre photographique. Mais les mutations successives n'ont pas perturbé l'équilibre du système, sa viabilité et la permanence de ses progrès. Les transitions ont eu lieu par glissements successifs, un choix en matière de procédés sensibles se substituant à un autre, sans heurt apparent ni cassure, jusqu'à la mise au point du procédé au gélatino-bromure d'argent. Des procédés chimiques différents ont été simultanément utilisés, mais souvent pour des usages distincts (portrait, paysage, applications scientifiques ou industrielles). Les recherches se sont effectuées par chevauchement entre les différentes techniques. Les questions relatives aux supports (plaque métallique, papier, verre, celluloïd) sont liées aux problématiques concernant les procédés chimiques (daguerréotype, albumine, collodion, gélatino-bromure d'argent). L'utilisation d'objectifs de combinaison optique différente (achromatiques, aplanétiques, anastigmatiques) coexiste durant une certaine période, malgré la révolution provoquée par la mise au point des nouveaux verres d'léna. Des systèmes d'obturation mettant en scène des matériaux (bois, métal) et des 
mécanismes de nature différente (volet, guillotine, rideau, obturateur central, obturateur de plaque) et ayant des degrés de précision divers sont envisagés au même moment.

Le procédé au gélatino-bromure d'argent : point d'équilibre du système

Deux périodes apparaissent très nettement (1839-1880 et 1880-1920) laissant supposer une discontinuité périodique dans la dynamique du système. La première période est marquée par l'acheminement progressif vers la mise au point de l'émulsion au gélatino-bromure d'argent.

L'espoir d'accéder à l'instantanéité, sans subir les inconvénients pratiques du procédé au collodion humide, résidait dans l'usage de la gélatine, dont Louis Alphonse Poitevin ${ }^{14}$ en préconisa l'utilisation en photographie, à l'occasion de l'une des séances de l'Académie des sciences. La gélatine fut de nouveau proposée pour entrer dans la composition de diverses formules. Hock déposait, par exemple, en 1867 un brevet ( $n^{\circ} 74881$ ) pour un « produit par le collodion combiné avec de la gélatine ॥. En 1871, Richard Leach Maddox faisait part de ses essais sur la gélatine bromurée, au sein du British Journal of photography ${ }^{15}$. II s'agissait, selon Désiré van Monckhoven, « du premier effort sérieux pour faire une émulsion à la gélatine $\|^{16}$.

L'avantage de cette émulsion, fondée sur l'emploi d'une mince couche de gélatine dans laquelle était emprisonné un sel sensible d'argent insoluble, était considérable. Nombre de scientifiques et de photographes, d'amateurs contemporains voyaient dans ce procédé une révolution dans la technique photographique. La facilité qu'il offrait d'être employé à sec et l'accès à l'instantané en faisaient le procédé de l'avenir. Encore nous faut-il y mettre un bémol. Ce phénomène autour de l'instantané, affirmé et encouragé dans les multiples manuels et traités de photographie, contemporains à cette découverte, est à relativiser dans la pratique réelle. Par faute de moyens techniques adéquats, ou de savoir-faire effectif, l'accès à l'instantanéité fut sans doute loin d'être immédiat.

14 Louis Alphonse Poitevin, « Nouveaux développements de la photographie sur gélatine "), La Lumière, 1851, Marseille, rééd. Jeanne Laffitte, 1996, 2 vol, pp. 89-90.

15 Richard Leach Maddox, séance du 10 novembre 1871 de la Société française de Photographie, Bulletin de la Société française de photographie, 1871, Paris, Société française de photographie, pp. 271-274.

16 Désiré van Monckhoven, Traité général de photographie, suivi d'un chapitre spécial sur le gélatino-bromure d'argent Paris, G. Masson, 1880, p. 375.
Mais les propriétés de l'émulsion furent à l'origine d'une aisance nouvelle du point de vue du matériel photographique. Plus besoin de tente, de matériel encombrant et lourd, une chambre noire, quelques glaces et le photographe était prêt à opérer. La vulgarisation de la pratique de la photographie résidait dans la simplification de sa technique. Ce procédé donna un nouvel essor à la photographie et autorisa l'industrialisation de cette filière (la production à grande échelle des surfaces sensibles). Impliquant l'accessibilité nouvelle de la technique, il provoqua l'apparition des appareils à main, et suscita un intérêt rapide et croissant des fabricants de 1885 à 1905.

Les recherches relatives à l'élaboration des procédés sensibles, empruntant à la chimie organique puis à la chimie de synthèse, restent majeures au cours de la période 1880-1920. Cependant, à partir de l'élaboration du procédé au gélatino-bromure d'argent, ces recherches visent moins à l'augmentation de la rapidité de l'émulsion qu'à gagner en sensibilité chromatique, développant par là les possibilités reproductives de la photographie, ses formes et ses applications scientifiques et industrielles (certes déjà bien ancrées sous le Second Empire).

La lutte contre les aberrations a constitué une constante dans les progrès de l'optique. Les exigences des opérateurs se précisèrent, notamment en fonction des usages. Au début des années 1880, Otto Schott et Ernest Abbe remplacèrent la chaux, qui entrait normalement dans la composition des verres d'optique, par des doses de baryte et d'oxyde de zinc, jointes à d'autres substances (didyme, urane, bore, manganèse, arsenic), appliquant ainsi les théories posées quarante ans précédemment par Joseph Max Petzval. Le nouvel objectif de Carl Zeiss, dit "anastigmat", a été présenté à la Société française de photographie par Charles Fabre, en $1892^{17}$. La construction des objectifs anastigmatiques constitua un progrès capital, puisqu'ils permettaient désormais de travailler avec de grandes ouvertures, tout en donnant une netteté sur toute l'étendue du sujet. Arrivant en même temps que l'augmentation de sensibilité des plaques photographiques, ces nouveaux objectifs marquèrent un pas décisif dans la question de l'instantanéité.

Mais l'influence de la découverte du gélatinobromure d'argent est majeure ce qui concerne les systèmes d'obturation, quasiment inexistants, puisque

17 Charles Fabre, « Présentation d'un nouvel objectif de Carl Zeiss dit Anastigmat ", Compte rendu de la séance du 8 janvier 1892 de la Société française de photographie, Bulletin de la Société française de photographie, Paris, Société française de photographie, 1892, pp. 44 et 71-72. 


\section{L'évolution des techniques photographiques}

sans raison d'être, jusqu'à ce surcroît de sensibilité des émulsions. Nombre de systèmes, empruntant à la mécanique de précision, sont commercialisés.

L'évolution des châssis n'est en liaison directe avec celle des procédés sensibles qu'en ce qui concerne l'adaptation au support utilisé pour les recevoir (cuivre, papier, verre, celluloïd). Le châssis s'adapte aux spécificités physiques des matériaux (résistance à la traction et à la pression, flexibilité, manipulations...) et aux aspirations du photographe. Ainsi, dans un souci pratique, les châssis à magasin et à escamotage, permettant de contenir et d'exposer successivement une douzaine vues, équipèrent les appareils à main.

\section{L'industrialisation de la filière photographique}

Les performances des appareils photographiques se sont particulièrement accrues au cours de cette période. Les constructeurs ont répondu à la demande des praticiens en proposant un éventail de produits s'échelonnant de l'appareil de base (le plus accessible selon un critère économique) à l'appareil perfectionné, en passant par l'appareil de milieu de gamme (qui représenta l'essentiel de la production). Le choix du consommateur se faisait également en fonction de l'usage, qui allait motiver et conditionner sa pratique de la photographie. On pourra ajouter ce critère social, le désir de distinction, qui anima certains amateurs. Le degré de performance d'un appareil photographique peut cependant paraître aléatoire si l'on se réfère à une pratique générale de la photographie. Un appareil de type « détective », muni d'un objectif rectilinéaire et d'un obturateur à guillotine, sera d'un usage limité par rapport à un « folding », pourvu d'un objectif anastigmatique et d'un obturateur central, mais dans la plupart des cas, les résultats seront comparables.

Un style technologique nouveau, caractérisé par l'usage bientôt systématique du métal, par le volume réduit, et l'intégration des éléments (châssis ou boîte à escamoter, optique, obturateur) au boîtier, se substitue au précédent, marqué par l'emploi du bois, le volume important, et la combinaison des éléments. L'intégration de l'objectif, de l'obturateur et du châssis au boîtier de l'appareil n'exclut pas l'interchangeabilité de ces éléments. L'opérateur pouvait adapter son appareil aux impératifs de la prise de vue. L'appareil a gagné en compacité. Le métal, entrevu au cours de la première période ${ }^{18}$, entre dans la construction des

18 Le constructeur anglais Arthur James Melluish avait proposé en 1860 l'emploi des métaux dans la construction des chambres noires, alors que l'on avait usé jusque-là que du bois. II déposa un brevet, en Angleterre, le 10 novembre 1859 pour une chambre photographique en laiton ou chambres photographiques, ne se limitant plus aux seuls systèmes optiques (montures des objectifs) et ferrures. La chambre photographique regroupe des typologies nouvelles, dont certaines n'ont été jusqu'alors qu'entrevues. Citons la Chambre automatique d'Adolphe Bertsch ou la Jumelle d'Octave Nicour On distingue les « détectives », les « jumelles », les « klapp », les " box ", les "folding ", les chambres d'atelier et les chambres de "touriste». On parle d'ailleurs plus volontiers d'appareils photographiques, notion moins restrictive et adaptée à ce nouveau paysage, pour désigner l'instrument de la photographie. Le vocable de chambre photographique ne désigne désormais plus que les appareils munis d'un soufflet, et dont l'emploi nécessite l'usage d'un pied (chambres d'atelier ou chambres de touriste). L'appareil photographique d'amateur est restreint, maniable. En même temps, ses performances techniques augmentent constamment, offrant à l'utilisateur une gamme de possibilités (qui ne garantissent pourtant ni la réussite, ni la qualité de l'opération).

Ce style technologique résulte certes de l'évoIution du système mais également des normes établies par les congrès de photographie successifs, et notamment par le Congrès de photographie de Paris, qui se tint en 1889 dans le cadre de l'exposition universelle. Ce congrès détermina ainsi des « dimensions normales " pour les vis des pieds des chambres noires, les montures des objectifs et leurs planchettes, les papiers et plaques sensibles. La Société française de photographie, déférant aux vœux formulés par ce dernier, fit exécuter des instruments de vérification, ou des pièces types, afin de permettre aux constructeurs comme aux amateurs de vérifier la conformité du matériel avec les dimensions et dispositions prescrites. Outre ces décisions, les congrès abordèrent d'autres aspects, techniques ou législatifs, liés à l'expression des

en cuivre argenté. Selon Charles Fabre, il avait démontré " qu'un appareil en acajou qui pèserait seize livres n'en pèserait que douze s'il était en laiton, et trois seulement s'il était en aluminium ॥. Arthur James Melluish, séance du 18 mai 1860 de la Société française de photographie, Bulletin de la Société française de photographie, 1860, Paris, Société française de photographie, pp. 166-167; Charles Fabre, Traité encyclopédique de photographie, tome I, Paris, Gauthier-Villars \& Fils, 1889, p. 236. Auguste Adolphe Bertsch concevait « un appareil de voyage pour l'obtention de petits clichés propres aux grossissements ", ou "chambre automatique ", en métal. Auguste Adolphe Bertsch, séance du 20 juillet 1860 de la Société française de photographie, Bulletin de la Société française de photographie, 1860, Paris, Société française de photographie, pp. 204208 ; « Nouvelle chambre noire de M. Bertsch », La Lumière, 1860, pp. 129-130, Marseille, réed. Jeannne Laffitte, 1996. 
formules photographiques, au problème des dénominations photographiques, aux formalités de douanes pour la circulation des préparations sensibles à la lumière, ou à la protection de la propriété des œuvres photographiques.

Le système technique photographique a ainsi généré deux types de structures de production et de consommation de 1839 à 1920. La première période correspond à la mise en place de structures liées à l'apparition de la technique nouvelle. Les structures de production et de consommation se sont organisées autour d'îlots préexistants, et correspondant à des techniques préexistantes (optique, chimie, ébénisterie). Puis le système a acquis une dimension industrielle et a acquis son autonomie. On assista à la multiplication des produits nouveaux, évolution sensible au niveau des dépôts de brevets et de marques. L'offre s'est accrue considérablement, et parut répondre à la demande latente.

La période s'écoulant entre les phases d'invention et d'innovation se réduit. Les produits furent plus rapidement absorbés par le marché. Ces quelques exemples le démontrent. Brevetée le 13 mars 1863, la chambre automatique d'Auguste Adolphe Bertsch ne fut présentée à la Société française de photographie que le 5 août 1864 (il fallut dix-sept mois pour que le brevet, qui marque l'invention, soit diffusé). Le Dubroni de Jules Bourdin fit l'objet d'un brevet le 15 mai 1864 ; il fut présenté à la Société française de hotographie le 21 avril 1865 (soit onze mois après le dépôt) ; il est mentionné dans le volume de L'Année scientifique et industrielle, publié par Louis Figuier, faisant état des nouveautés techniques de l'année 1865. II ne faut plus que huit moins entre le dépôt du brevet et la présentation à la Société française de photographie pour le Vérascope de Jules Richard (30 octobre 1893 et 1 1 juin 1894), le Carnet Photo Rapide de Charles Victor Le Roy (17 avril 1888 et 7 décembre 1888) et l'Escopette d'Albert Darier ( 13 mai 1889 et 3 janvier 1890).

Vers 1904, le marché semble saturé. Les dépôts de brevets diminuent de façon conséquente. On peut proposer une hypothèse économique et une hypothèse technique. L'offre apparaît supérieure à la demande. Le marché n'est plus en mesure d'absorber les nouveautés. Mais on peut aussi penser que ce blocage du système provient de ce que toutes les innovations, qui reposent sur le gélatino-bromure d'argent, ont été exploitées. Cette crise conjoncturelle cessa rapidement. La dynamique reprit à partir de 1906 , mais selon un rythme moins soutenu, au moment de l'apparition et la commercialisation du procédé de photographie en couleurs, l'autochrome, d'Auguste et Louis Lumière.
La dynamique du système, révélée par la pratique de la photographie, a été confortée par des facteurs annexes : la demande de la science et de l'industrie, mais également le développement du tourisme. La science et l'industrie se sont, dès 1839, posées en demandeurs par rapport au système photographique en imposant des exigences, qui ont joué un rôle moteur (développement d'une technologie pour satisfaire une application).

Ainsi, l'élaboration de l'obturateur à guillotine par Armand Hippolyte Louis Fizeau et Jean Bernard Léon Foucault en 1845 et l'obtention d'images du Soleil au daguerréotype au 1/60ème de seconde apparaît comme un dysfonctionnement du système technique photographique. L'élaboration de cet obturateur - et l'apparition effective de cet appareil en tant que dispositif autonome au moment ou l'obturation est encore assuré par le bouchon d'objectif - marque une avance de près de tente cinq années sur le système technique photographique. La science devient le moteur de l'évolution du système technique photographique. Les recherches sur l'orthochromatisme sont motivées par l'application de la photographie à la météorologie (photographie des nuages) ou à la micrographie (photographie des préparations histologiques). La médecine est à l'origine de la chronophotographie et de son développement. L'élaboration, en 1887, dans un but médical requérant l'usage de la photographie instantanée, de la « chambre portative à prisme pour photographie instantanée ") d'Albert Londe et Charles Dessoudeix, permettant « de viser le modèle, de le mettre au point et de le saisir immédiatement sans temps perdu aucun ", préfigure l'adoption de la visée reflex adoptée tardivement par les constructeurs.

Le schéma se profile différemment quant aux applications industrielles de la photographie. L'apport de l'industrie envers le système technique photographique se résume essentiellement aux supports. On se concentra sur les moyens de concilier image photographique et produits de l'industrie. On appliqua les photographies sur les étoffes, le verre, la faïence ou l'acier. II n'y eut pas d'évolution parallèle entre les deux systèmes, mais transfert et adaptation du système technique photographique vers l'industrie.

Le développement de l'activité touristique, subséquent à celui des transports, le chemin de fer, en ce qui concerne le tourisme lointain, et le vélocipède, pour le tourisme de proximité, est survenu d'une manière concomitante à la vulgarisation de la pratique de la photographie. Dans les deux cas, les structures économiques et l'évolution des mentalités, se sont révélées favorables à ce phénomène. Tourisme et 


\section{L'évolution des techniques photographiques}

photographie se découvrirent un intérêt commun et réciproque. La photographie était en mesure de servir le tourisme par la diffusion d'images des sites et monuments. Le tourisme pouvait s'associer à la photographie, et en devenir un élément moteur. Cette pratique fut encouragée par la Société d'excursions des amateurs de photographie (1887) ou l'Association des amateurs photographes du touring-club de France (1879). Dans le cas du tourisme de proximité, la bicyclette fut le vecteur de cette association. La stéréoscopie et la projection n'en constituaient pas moins des attraits, qui ne pouvaient qu'inciter les amateurs à pratiquer le tourisme. La carte postale consacra également cette activité.

\section{Publicité et transmission des savoirs}

La transmission des savoirs, le passage de l'invention à l'innovation, s'effectua par le biais d'un dialogue, réciproque, entre inventeur, vulgarisateur, entrepreneur et consommateur.

Les effectifs de la Société française de photographie ont été en constante augmentation (en exceptant les périodes de crises politiques, soit les conflits de 1870-1870 et de 1914-1918). Mais il faut dégager, parmi les membres, les éléments moteurs des éléments périphériques, qui bénéficiaient des progrès sans y contribuer. La présentation d'un procédé ou d'un appareil devant les membres de la Société légitimait l'invention. Le Bulletin de la Société française de photographie constitua ainsi une sorte de vivier dans lequel venaient puiser les vulgarisateurs scientifiques. Citons parmi les vulgarisateurs, membres de surcroît de la Société française de photographie, Lovis Alphonse Davanne, Albert Londe ou Léon Vidal, qui publièrent un nombre conséquent de traités portant sur la pratique générale ou sur des applications spéciales de la photographie. II est fréquent de découvrir, au fil des dépouillements de revues ou de bulletins d'association, des communications présentées antérieurement à la Société française de photographie. Notons que la société mit en place une politique d'échange de publications avec d'autres sociétés savantes. Le monde photographique dans son ensemble gravitait ainsi autour de la Société française de photographie, établissant des liens plus ou moins directs avec cette institution. Les fabricants prirent, à partir des années 1880, le relais des amateurs qui constituaient la majorité des membres de la société depuis sa création. Les inventeurs se cachaient désormais derrière une marque de fabrique. La phase d'industrialisation eut pour conséquence une diminution conséquente des recherches menées par les amateurs éclairés. Ce phénomène est visible au sein du Bulletin de la Société française de photographie mais aussi au niveau des dépôts de brevets.

Mais le choix de publier un ouvrage, de déposer un brevet, une marque ou de communiquer dans une société savante fait apparaître, chez les constructeurs, des stratégies différentes du point de vue de la protection de l'invention mais aussi de sa diffusion.

Léon Gaumont déposa plus d'une trentaine de brevets ; les frères Lumière en déposèrent près d'une quarantaine; Henri Mackenstein en déposa une vingtaine ; George Eastman en déposa près de cinquante. Si la plupart des constructeurs choisissent l'une ou l'autre protection, demande de brevet ou de marque, certains, tel Léon Gaumont, n'hésitent pas à entreprendre simultanément les deux démarches pour une même invention ${ }^{19}$. Jules Richard privilégie le dépôt de brevet: il en dépose plus d'une soixantaine, pour une quinzaine de marques et fait seulement trois interventions à la Société française de photographie. D'autres, comme Félix Maxime Richard, la société de George Eastman, ou Léon Gaumont, se font une spécialité de déposer régulièrement des listes de noms (orthographes différentes pour un même nom ; noms différents), dont la plupart restèrent orphelins. Félix Maxime Richard comprend l'impact du nom « Kodak " ${ }^{20}$ et dépose dès l'année 1895 les noms «Phodak » et «Phodek » 21, qui restèrent cependant à l'état de projets.

Les communications faites au sein de la Société française de photographie font apparaître d'autres manœuvres commerciales, des stratégies de valorisation reposant parfois sur des accords entre constructeurs. Félix Maxime Richard effectue plus d'une trentaine de dépôts de marques, dont les noms «Photo-Jumelle " ${ }^{22}$

19 La société Léon Gaumont et Compagnie dépose un brevet (n³22894) en 1902 pour un « Appareil photographique réductible dénommé Block-Notes " (certif. add. n¹989 en 1903) et n³404 en 1904) et une marque la même année ( $n^{\circ} 076516$ ) pour désigner des « appareils photographiques réductibles dénommés: Block-Notes $»$.

20 Dépôt de marque n004961, 04/03/1890, The Eastman Photographic Materials Company Limited, Londres (Grande-Bretagne).

21 Dépôts de marque n046284 et 046285, 16/02/1895, Félix Maxime Richard, ingénieur constructeur, fabricant de fournitures pour la photographie, Paris.

22 Jules Carpentier dépose en 1892 la marque « Photo-Jumelle » ( $n^{\circ} 038596$ ) pour désigner « des appareils photographiques dits photo-jumelles ॥. II renouvelle le dépôt en 1907 (n¹01 198). Félix Maxime Richard dépose en 1894 une marque ( $n^{\circ 043395)}$ pour désigner « des boîtes, paquets ou emballages contenant une ou plusieurs charges de dix-huit plaques pour photo-jumelle à répétition », où apparaît la mention « Paquetage par 18 plaques spécial pour Photo-Jumelle à Répétition, Comptoir Général de Photographie, Paris ». L'année suivante, il dépose 
et «Spido " ${ }^{23}$ correspondant à des appareils photographiques produits et proposés à la vente au Comptoir général de photographie, où l'on retrouve Jules Carpentier et Léon Gaumont. Notons qu'il intervient à une quinzaine de reprises au sein de la Société française de photographie ; Jules Richard, son frère et concurrent sur ce marché, ne fera que trois interventions. Léon Gaumont en compte une cinquantaine à son actif.

Les noms des inventeurs ou les marques de fabrique étaient en général associés à un type d'objet. D'une manière générale, on peut parler de spécialisation d'un type de production. Cette spécialisation résultait de compétences particulières ou de l'intérêt particulier des fabricants mais aussi des spécificités de l'appareil de production lorsque ces derniers étaient déjà engagés dans un processus industriel (Jules Carpentier et Jules Richard sont familiers de la mécanique de précision). Les noms de Jules Carpentier, d'Henri Mackenstein, de Léon Gaumont et d'Henri Bellieni sont liés à l'apparition et à l'essor des jumelles photographiques; celui de Jules Richard consacre la photographie stéréoscopique par les appareils à main ; ceux d'Auguste et de Louis Lumière sont associés aux surfaces sensibles. L'opticien J. Fleury-Hermagis proposait quant à lui objectifs et appareils ("Vélocigraphe », « Vélo-Jumelle »).

Seule la firme Eastman Kodak paraît contrôler l'intégralité de la chaîne de prise de vue mais également le laboratoire: produits chimiques, surfaces sensibles, appareil à main, développement et tirage. Elle propose les produits mais également les services. La société assure le développement et le tirage des vues. George Eastman préfigura l'évolution qui fut celle de la photographie professionnelle : le photographe professionnel élargit son activité en se voyant bientôt confier par l'amateur les opérations de laboratoire. Eastman écrivait en 1888 dans la notice du Kodak :

" The principle of the Kodak system is the separation of the work that any person whomsoever can

une marque (n046976), où la mention "Comptoir Général de Photographie " est inscrite sur une représentation de face de la Photo-Jumelle. En 1897, Léon Gaumont dépose une marque ( $n^{\circ} 054970$ ) pour désigner des « tarifs, prospectus, catalogues et annonces pour la photographie " : étoile avec inscription « L. G. \& Cie » et dessin de la Photo-Jumelle sur laquelle est inscrit « Comptoir Gal de Photographie ». Ce dépôt est renouvelé en 1914 par la Société des Etablissements Gaumont (marque n 157764 : étoile à douze branches « L. G \& Compagnie Paris ॥, Photo-Jumelle de face avec l'inscription « Comptoir Gal de Photographie »).

23 En 1894, Félix Maxime Richard dépose la marque «Spido » pour désigner des articles photographiques ( $\left.n^{\circ} 043113\right)$. do in making a photograph, from the work that only an expert can do ${ }^{24}$.

II ajoutait :

" With the Kodak, we furnish anybody, man, woman or child, who has sufficient intelligence to point a box straight and press a button with an instrument which altogether removes from the practice of photography the necessity for exceptional facilities, or in fact, any special knowledge of the art. It can be employed without preliminary study, without a dark room, and without chemicals $1{ }^{25}$.

George Eastman fait surtout figure d'innovateur en terme de marketing. Mais n'oublions pas qu'en 1874, dans la notice du Scénographe, Candèze incitait l'amateur à se procurer des glaces au collodion sec chez Deyrolle, à Paris, constructeur de l'appareil, et à confier le développement et le tirage des vues à un photographe professionnel :

"Les vues prises, on les livrera à un photographe qui les développera, en tirera des épreuves sur papier, exécutera enfin toutes les manipulations délicates qui réclament une certaine habitude des opérations photographiques 1 .

La notice était du reste divisée en deux parties distinctes :

" La première servira de guide à l'amateur qui achètera ses glaces toutes préparées et fera tirer les épreuves par un homme de métier, et qui n'a donc besoin que d'être initié à l'emploi du Scénographe; la seconde ne sera utile qu'aux personnes qui voudront s'exercer dans l'art du photographe et exécuter elles-mêmes toutes les manipulations nécessaires [" préparation des glaces sensibles, leur développement définitif après

24 « Le principe du système Kodak est fondé sur la distinction entre le travail que toute personne, quelle qu'elle soit, peut effectuer en faisant une photographie, et celui que seul un expert peut exécuter $»$.

25 « Avec le Kodak, nous fournissons quiconque, homme, femme ou enfant, qui a suffisamment d'intelligence pour orienter une boîte vers l'avant et d'appuyer sur un bouton avec un instrument qui supprime de la pratique de la photographie la nécessité d'équipements exceptionnels, ou en fait, n'importe quelle connaissance spéciale de cet art. Il peut être utilisé sans étude préliminaire, sans salle obscure, et sans produits chimiques 1 . 


\section{L'évolution des techniques photographiques}

l'exposition ammoniacale, enfin le tirage des épreuves sur papier ou sur verre ॥] 1.

Candèze précisait, reprenant les arguments développés par Jules Bourdin, concepteur de la chambre-laboratoire dite Dubroni, quelques années auparavant :

"Ceci s'adresse surtout aux dames, que rebute l'inconvénient de se tacher les doigts dans le maniement des liquides employés en photographie, et puis aux personnes auxquelles le temps fait défaut pour s'exercer dans la pratique de cet art et mener à bien les opérations chimiques [...]. »

Même le slogan du Scénographe ressemblait à celui du Kodak:

" Avec cet appareil chacun peut faire de la photographie sans jamais avoir appris ».

\section{Conclusion}

Le choix et l'exploitation de ce corpus de sources inédites, et la méthodologie développée dans ce cadre, ont permis de dégager des paramètres expliquant la dynamique de l'innovation appliquée à la filière photographique. La chimie constitua le moteur de l'innovation en photographie. Le procédé au collodion humide fut un premier progrès en matière d'instantanéité. Les procédés à l'albumine et au collodion sec facilitèrent le protocole de la prise de vue. En autorisant l'industrialisation de la filière, le procédé au gélatino-bromure d'argent fit entrer la photographie dans une économie de marché, où les stratégies déployées par les inventeurs et les fabricants prennent sens sur les plans concurrentiel et commercial. L'amateur, éclairé ou néophyte, eut un rôle primordial dans l'évolution des techniques photographiques. Dans un premier temps, se plaçant en inventeur, il est à l'origine des progrès de la photographie ; puis, en position de consommateur, il soutient l'innovation. Les outils (communications, publications) et les lieux (cercles savants) de la transmission des savoirs ont ainsi été identifiés. Ils recoupent les stratégies de valorisation et de promotion développées par l'inventeur : communications dans une société savante, publications, dépôts de brevets et/ou de marque.

Enfin, ce modèle a été appliqué aux sources objets. Il a formé un élément de compréhension important dans l'étude de la constitution des collections photographiques (objets en grandeur réelle, modèles, épreuves) du musée du Conservatoire des arts et métiers. ॥ a permis d'expliquer la périodisation et le volume des acquisitions du point de vue du contexte technique de l'invention. Certes, le développement des acquisitions dans ce domaine, au début des années 1880, est lié à la volonté de l'établissement de créer un espace d'exposition dédié à la photographie et aux arts graphiques. Mais cette décision, tardive si l'on considère la date de divulgation du procédé daguerréotype, ne peut être dissociée de l'essor des applications de la photographie et de la vulgarisation de sa pratique, caractérisée par l'explosion des dépôts de brevets, à cette période. Le point fort de la constitution de cette collection se situe ainsi dans la phase d'industrialisation de la photographie ; ce qui pose la question de la défaillance du Conservatoire en matière de veille technologique. Ajoutons que la collection s' accroît en relation étroite avec la Société française de photographie, et ses membres - notamment Louis Alphonse Davanne, membre fondateur et président de 1876 à 1901 ; ce qui renforce le choix de nous attacher à La Lumière puis au Bulletin de la Société française de photographie en tant que filtres de l'innovation. 